\title{
Brain-derived neurotrophic factor (BDNF) and type 2 diabetes
}

\author{
K. S. Krabbe • A. R. Nielsen • R. Krogh-Madsen • \\ P. Plomgaard - P. Rasmussen - C. Erikstrup • \\ C. P. Fischer • B. Lindegaard • A. M. W. Petersen • \\ S. Taudorf $\bullet$ N. H. Secher $\cdot$ H. Pilegaard . \\ H. Bruunsgaard $\cdot$ B. K. Pedersen
}

Received: 27 June 2006 / Accepted: 19 October 2006 / Published online: 7 December 2006

(C) Springer-Verlag 2006

\begin{abstract}
Aims/hypothesis Decreased levels of brain-derived neurotrophic factor (BDNF) have been implicated in the pathogenesis of Alzheimer's disease and depression. These disorders are associated with type 2 diabetes, and animal models suggest that BDNF plays a role in insulin resistance. We therefore explored whether BDNF plays a role in human glucose metabolism.

Subjects and methods We included (Study 1) 233 humans divided into four groups depending on presence or absence of type 2 diabetes and presence or absence of obesity; and (Study 2) seven healthy volunteers who underwent both a hyperglycaemic and a hyperinsulinaemic-euglycaemic clamp.

Results Plasma levels of BDNF in Study 1 were decreased in humans with type 2 diabetes independently of obesity. Plasma BDNF was inversely associated with fasting plasma glucose, but not with insulin. No association was found
\end{abstract}

K. S. Krabbe $(\bowtie) \cdot$ A. R. Nielsen $\cdot$ R. Krogh-Madsen •

P. Plomgaard $\cdot$ C. Erikstrup $\cdot$ C. P. Fischer $\cdot$ B. Lindegaard $\cdot$

A. M. W. Petersen $\cdot$ S. Taudorf $\cdot H$. Pilegaard $\cdot H$. Bruunsgaard

B. K. Pedersen

The Centre of Inflammation and Metabolism,

Department of Infectious Diseases, Faculty of Health Sciences,

University of Copenhagen,

Copenhagen, Denmark

e-mail: karen.krabbe@dadlnet.dk

P. Rasmussen • N. H. Secher $\cdot$ H. Pilegaard - B. K. Pedersen

The Copenhagen Muscle Research Centre, Rigshospitalet,

Faculty of Health Sciences, University of Copenhagen,

Copenhagen, Denmark

\section{H. Pilegaard}

Institute of Molecular Biology and Physiology,

University of Copenhagen,

Copenhagen, Denmark between the BDNF G196A (Val66Met) polymorphism and diabetes or obesity. In Study 2 an output of BDNF from the human brain was detected at basal conditions. This output was inhibited when blood glucose levels were elevated. In contrast, when plasma insulin was increased while maintaining normal blood glucose, the cerebral output of BDNF was not inhibited, indicating that high levels of glucose, but not insulin, inhibit the output of BDNF from the human brain. Conclusions/interpretation Low levels of BDNF accompany impaired glucose metabolism. Decreased BDNF may be a pathogenetic factor involved not only in dementia and depression, but also in type 2 diabetes, potentially explaining the clustering of these conditions in epidemiological studies.

Keywords Brain · Glucose · Insulin · Neurotrophin ·

Polymorphism

$\begin{array}{ll}\text { Abbreviations } \\ B D N F & \text { brain-derived neurotrophic factor } \\ C R P & \text { C-reactive protein } \\ \text { CVD } & \text { cardiovascular disease } \\ \text { HOMA2- } & \text { homeostatic model assessment (version 2) for } \\ I R & \text { insulin resistance } \\ \text { SNP } & \text { single nucleotide polymorphism }\end{array}$

\section{Introduction}

In this paper we focus on the possibility that brain-derived neurotrophic factor (BDNF) may, in addition to its role in neuronal health, play a systemic role in human glucose metabolism. 
BDNF is a member of the neurotrophic factor family, which plays a key role in regulating survival, growth, and maintenance of neurons [1]. In addition, BDNF plays a role in learning and memory (for review see [2]). It has been suggested that decreased production of BDNF is a pathogenetic factor common to Alzheimer's disease and major depression, which might explain the association between the two disorders [3]. Hippocampal samples from Alzheimer's disease donors show decreased BDNF expression [4], and individuals with Alzheimer's disease have low plasma levels of BDNF [5]. In addition, major depression patients have lower levels of serum BDNF than normal control subjects [6].

In animals BDNF is involved in insulin resistance. BDNF reduces food intake and lowers blood glucose levels in obese diabetic mice [7-9]. The hypoglycaemic effect of BDNF cannot be ascribed solely to the hypophagic effect of BDNF, because BDNF administration has beneficial effects on glucose homeostasis and improves insulin resistance in $d b / d b$ mice even when food-intake is controlled [7-9]. The role of BDNF in metabolism is supported by studies on BDNF-deficient mice $\left(\mathrm{Bdnf}^{+/-}\right.$), which develop hyperphagia and obesity in early adulthood [10]. However, when BDNF is administered to normal mice or rats, it has no effect on blood glucose levels, indicating that BDNF exerts its effects by enhancing insulin sensitivity [7]. Further, BDNF activates several signalling pathways also activated by insulin, including phosphatidylinositol-3 kinase/Akt among others (reviewed in [11]).

In humans, type 2 diabetes is associated with impaired cognitive function, including learning, memory, and processing speed $[12,13]$. Large longitudinal population-based studies show that the rate of cognitive decline is accelerated in elderly people with type 2 diabetes [14]. A recent review [15] showed that the incidence of 'any dementia' was higher in individuals with type 2 diabetes than in those without. This high risk included both Alzheimer's disease and vascular dementia. Individuals with type 2 diabetes also have a high prevalence of affective illness, $~ 11$ to $15 \%$ meeting the criteria for major depression [16]. Vascular disease and alterations in glucose, insulin, and amyloid metabolism may be underlying mechanisms that explain the link between diabetes and neuropsychiatric diseases [15, 17-19]. A causal relationship between type 2 diabetes and insulin resistance on the one hand, and cognitive impairment, dementia, and major depression on the other, has not yet been demonstrated.

We tested the hypothesis that patients with type 2 diabetes have low levels of BDNF in plasma and examined whether the cerebral output of BDNF in humans is regulated by plasma levels of glucose or insulin, or both. Further, we tested whether the BDNF G196A single nucleotide polymorphism (SNP), which results in an amino acid substitution (Val/Met) and affects intracellular packaging of pro-BDNF and secretion of BDNF [20], is a risk factor in diabetes or obesity.

\section{Subjects and methods}

Study 1: Plasma BDNF in patients with diabetes (epidemiological study)

Design and participant recruitment We used a crosssectional case-control design. Participants were divided into four distinct groups according to whether they had or had not been diagnosed with type 2 diabetes and according to BMI $\left(\geq 30\right.$ or $\left.<30 \mathrm{~kg} / \mathrm{m}^{2}\right)$. Participants $(n=233)$ were recruited by advertising in a local newspaper and information on type 2 diabetes diagnosis was based on oral information from each subject. To verify the diagnosis, the WHO diagnostic criteria for type 2 diabetes were used. Participants were carefully screened to isolate metabolic conditions other than type 2 diabetes that are known to influence body composition and the immune system. Exclusion criteria were: treatment with insulin, recent or ongoing infection, history of malignant disease, and known dementia. Participants were categorised as having cardiovascular disease (CVD) if they had claudication or at least one of the following diagnoses: cerebrovascular accident, angina pectoris, or prior coronary artery bypass graft or percutaneous transluminal coronary angioplasty. Current intake of antidepressive medication was registered.

Participants received oral and written information about the experimental procedures before giving their written informed consent. The study was approved by the Ethical Committee of Copenhagen and Frederiksberg Council (01-141/04).

Protocol Participants reported in the laboratory between 08.00 and $10.00 \mathrm{~h}$ after an overnight fast. They did not take any medication in the $24 \mathrm{~h}$ preceding the examination, and those with type 2 diabetes did not take hypoglycaemic medication for 1 week preceding the examination. A general health examination was performed. Sphygmomanometric measurement of brachial blood pressure was performed on the participants while resting in supine position. Blood samples were drawn from an antecubital vein. On the same day, an OGTT was performed.

Oral glucose tolerance test Blood samples were drawn before, and 1 and $2 \mathrm{~h}$ after drinking $500 \mathrm{ml}$ of water containing $75 \mathrm{~g}$ of dissolved glucose. The WHO diagnostic criteria were applied, i.e. NGT was defined as fasting venous plasma glucose $<7.0$ and venous plasma glucose $<7.8 \mathrm{mmol} / \mathrm{l} 2 \mathrm{~h}$ after the oral glucose load; IGT was 
defined as fasting venous plasma glucose $<7.0 \mathrm{mmol} / \mathrm{l}$ and venous plasma glucose between 7.8 and $11.0 \mathrm{mmol} / 12 \mathrm{~h}$ after the oral glucose load; type 2 diabetes was defined as fasting venous plasma glucose $>6.9 \mathrm{mmol} / 1$ or venous plasma glucose $>11.0 \mathrm{mmol} / \mathrm{l} 2 \mathrm{~h}$ after the oral glucose load.

Study 2: Regulation of BDNF release from the human brain (experimental study)

Participants Seven healthy men (mean age 26.7 [range 2334] years; mean BMI 23.7 [range 21.2-27.7] kg/m $\mathrm{m}^{2}$ ) were included, following provision of oral and written informed consent. Before the study, the subjects underwent a thorough clinical examination and blood samples were taken for evaluation of renal, hepatic and thyroid function, haemoglobin, white blood cell counts, electrolytes, plasma insulin and plasma glucose. All tests were normal. The study conformed to the Helsinki declaration and was approved by the Ethical Committee of Copenhagen and Frederiksberg Council (01 257245).

Study design Subjects underwent two separate trials at least 1 month apart in a randomised order. One trial comprised a steady-state hyperglycaemic clamp and the other a hyperinsulinaemic-euglycaemic clamp. On the study day, the subject reported to the laboratory after an overnight fast. A peripheral catheter was placed in an antecubital vein for infusion. Under local anaesthetic (lidocaine, $2 \%$ ), a catheter was placed retrograde with the Seldinger technique in the right internal jugular vein $(1.6 \mathrm{~mm}, 14$ gauge; ES-04706; Arrow International, Reading, PA, USA). Placement of the catheter was guided by an ultrasound image and the catheter was subsequently advanced to the bulb of the vein. A third catheter was placed in the brachial artery $(1.1 \mathrm{~mm}, 20$ gauge) of the non-dominant arm. Cerebral perfusion was evaluated via ultrasound Doppler sonography (Transcan; EME, Überlingen, Germany) in the middle cerebral artery. Depending on the position with the best signal:noise ratio, the proximal part of the middle cerebral artery was insonated at a depth of 40 to $60 \mathrm{~mm}$ from the temporal bone and the probe was secured with a headband. Following instrumentation, the subjects rested in a semi-supine position with the back and head supported at an angle of $\sim 45^{\circ}$ for $140 \mathrm{~min}$. Cerebral perfusion was evaluated and blood samples from the right internal jugular vein and the brachial artery were drawn at baseline, just before initiation of the clamps (time point $140 \mathrm{~min}$ ), at steady state conditions during the clamp (260 min, corresponding to $2 \mathrm{~h}$ ), and at the termination of the clamp (320 min, corresponding to $3 \mathrm{~h}$ ).

Hyperglycaemic clamp Glucose $(200 \mathrm{~g} / 1,000 \mathrm{ml})$ was infused intravenously for $3 \mathrm{~h}$ to maintain a blood glucose level of $15 \mathrm{mmol} / \mathrm{l}$. The rate was adjusted by a computercontrolled infusion pump according to arterial blood glucose levels. To maintain potassium levels corresponding to baseline values, isotonic saline with potassium $(51 \mathrm{mmol} / \mathrm{l})$ was infused continuously. Measurement of glucose and potassium concentrations was done every $5 \mathrm{~min}$ for the first hour and then every $10 \mathrm{~min}$.

Euglycaemic clamp Insulin (Actrapid, $100 \mathrm{IU} / \mathrm{ml}$; Novo Nordisk Insulin, Bagsvaerd, Denmark) was infused continuously for $3 \mathrm{~h}$ at $0.08 \mathrm{U} \mathrm{min} \mathrm{m}^{-1}$. Glucose $(200 \mathrm{~g} / 1,000 \mathrm{ml})$ was infused by a computer-controlled infusion pump at rates adjusted to maintain blood glucose at $5 \mathrm{mmol} / \mathrm{l}$. Isotonic saline with potassium as well as $1,000 \mathrm{ml}$ of isotonic saline were infused continuously during the study. Arterial blood was analysed at intervals of $5 \mathrm{~min}$ in the first hour and then every $10 \mathrm{~min}$.

Both studies: measurement of BDNF, insulin, glucose, lipids, C-reactive protein and insulin resistance

Blood samples were drawn into glass tubes containing EDTA, which were immediately spun at $3,500 \times g$ for $15 \mathrm{~min}$ at $4^{\circ} \mathrm{C}$. Plasma was isolated and stored at $-20^{\circ} \mathrm{C}$ (Study 1) or $-80^{\circ} \mathrm{C}$ (Study 2) until analysed. Plasma concentrations of BDNF were measured by ELISA (R\&D Systems, Minneapolis, MN, USA). After thawing, samples were centrifuged at $10,000 \times g$ for $10 \mathrm{~min}$ at $4^{\circ} \mathrm{C}$ for complete platelet removal. Samples were analysed in duplicate, and mean concentrations were calculated. In Study 2 there was no significant change in cerebral perfusion over time and between trials (data not shown); thus untransformed data were used.

Plasma levels of cholesterol, lipoprotein, triacylglycerol, C-reactive protein (CRP), glucose and insulin were measured using routine laboratory methods (Study 1). In Study 2, plasma levels of insulin were analysed by ELISA (Dako, Glostrup, Denmark). Plasma glucose and potassium concentrations in arterial blood were measured immediately on an EML 105 (Radiometer, Copenhagen, Denmark).

Based on the fasting plasma concentrations of glucose and insulin, the level of insulin resistance was calculated using the homeostatic model assessment version 2 (HOMA2-IR) from 1996 (available at http://www.dtu.ox. ac.uk/), since the original HOMA [21] tends to overestimate insulin resistance [22].

SNP analyses

DNA was extracted from whole blood that had been stored at $-20^{\circ} \mathrm{C}$ using a kit (QIAamp DNA Blood Midi; Qiagen, Hilden, Germany). The BDNF 196 G/A polymorphism (rs 
6265) was determined using fluorescence-based real-time PCR (ABI PRISM 7900 Sequence Detection System; Applied Biosystems, Foster City, CA, USA). A predeveloped assay (C_11592758_10; Applied Biosystems) was used, containing forward and reverse primers, as well as an FAM and a VIC-labelled Minor Groove Binding TaqMan probe representing each of the allele possibilities. PCR amplification was performed in a total reaction volume of $5 \mu \mathrm{l}$. The reaction mixture consisted of $1 \mu \mathrm{l}$ $0.4 \mu \mathrm{g} / \mu \mathrm{l} \mathrm{gDNA}$, primer and probe mix $(20 \times)$, nucleasefree water and $2 \times$ TaqMan Universal MasterMix (Applied Biosystems) containing AmpliTaq Gold DNA polymerase, AmpErase Uracil $N$-glycosylase, dNTPs with dUTP, ROX as passive reference and buffer components. Immediately after a regular PCR run (cycle profile: $50^{\circ} \mathrm{C}$ for $2 \mathrm{~min}+$ $95^{\circ} \mathrm{C}$ for $10 \mathrm{~min}+\left[95^{\circ} \mathrm{C}\right.$ for $15 \mathrm{~s}+60^{\circ} \mathrm{C}$ for $\left.1 \mathrm{~min}\right] \times 40$ cycles), an allelic discrimination run was performed allowing for discrimination between the allele composition of each sample. DNA was available for 222 subjects. We were unable to determine zygosity in four cases. Thus the effective sample size was 218 .

\section{Statistics}

General The assumption of normality was tested using a probability plot and a Kolmogorov-Smirnov test. If data were not normally distributed, logarithmical transformation was performed (BDNF, insulin, glucose, triacylglycerol, CRP, HOMA2-IR). $p<0.05$ was considered significant. All analyses were performed with SAS 9.1 (SAS Institute, Cary, NC, USA).

Epidemiological study We distinguished between three glycaemia groups: NGT, IGT, type 2 diabetes. Unless otherwise stated, analyses were run excluding IGT subjects. For a description of the cohort, we defined two subgroups (obese, non-obese) within each glycaemia group. If an ANOVA revealed significant differences between the six subgroups, post hoc $t$ tests (SAS: PROC TTEST) were used to determine whether each glycaemia/obesity group differed from the group with NGT and no obesity in terms of: age, sex, BMI, fasting glucose and lipids, CRP, CVD, intake of antidepressive medication and smoking status.

Multiple regression analyses (SAS: PROC GLM) were performed to identify whether diabetes, obesity, lipid profile, CRP, CVD status, intake of antidepressive medication, statins or fibrates, and the $B D N F$ G196A SNP could explain some of the variation in plasma levels of BDNF (dependent variable). Reduction of the model was performed with 0.1 as the significance limit. As model control, normality of residuals (histogram) and a plot of residuals against predicted values were assessed.
To address which component of the diabetes diagnosis was most strongly correlated with plasma BDNF levels, we used a linear regression analysis (SAS: PROC REG) with $\log (\mathrm{BDNF})$ as the dependent variable and the following covariates: fasting plasma glucose, fasting plasma insulin and obesity. Correlation coefficients were calculated using the SAS PROC CORR statement. There was no collinearity between plasma glucose and plasma insulin.

To study differences in genotype frequencies between groups the $\chi^{2}$ test was used.

Experimental study The jugular-to-arterial concentration differences were calculated. In order to determine whether there was a net efflux of BDNF across the brain, the AUC corresponding to the two pre-clamp measurements on each experimental day was determined. A paired $t$ test showed that the AUCs did not differ between experimental days $(p=0.2)$ and, therefore, means for each subject were calculated. To test whether the resulting AUC was different from zero a $t$ test was performed.

Time-course during clamps An ANOVA for repeated measures including all four time-points was used (model: jugular-to-arterial difference in BDNF = time; subject was included as a random effect).

\section{Results}

Plasma BDNF is decreased in patients with type 2 diabetes

The characteristics of the cohort are shown in Table 1 . Figure 1 illustrates that plasma levels of BDNF were decreased in diabetic participants relative to those without type 2 diabetes $(F=20.0, p<0.0001)$, and in obese relative to non-obese participants $(F=6.1, p=0.01)$. There was no interaction between diabetes and obesity status on plasma BDNF concentrations. These associations remained unchanged when adjusted for age, sex and smoking status, which had no effect on plasma BDNF levels $\left(R^{2}\right.$ for the model: 13\%). Inclusion of subjects with IGT as a third glycaemia group demonstrated the same association pattern $(p<0.0001)$. The effect of obesity was unchanged $(p=0.02)$.

Diabetes and obesity retained their effects in a multivariate analysis including CVD, hypertension, cholesterol, CRP, BDNF G196A zygosity and intake of antidepressive medication, statins or fibrates along with age, sex and smoking status ( $R^{2}$ for the model: $25 \%$ ). Furthermore, this analysis showed no effect on plasma BDNF of CVD, hypertension, age, sex, smoking status, intake of antidepressive medication, statins or fibrates or BDNF G196A 
Table 1 Characteristics of the study population (Study 1)

\begin{tabular}{|c|c|c|c|c|c|c|c|}
\hline Variable & $\begin{array}{l}\text { NGT, } \\
\text { non-obese }\end{array}$ & NGT, obese & $\begin{array}{l}\text { IGT, } \\
\text { non-obese }\end{array}$ & IGT, obese & $\begin{array}{l}\text { Diabetes, } \\
\text { non-obese }\end{array}$ & $\begin{array}{l}\text { Diabetes, } \\
\text { obese }\end{array}$ & $\begin{array}{l}p \text { value } \\
\text { for ANOVA }\end{array}$ \\
\hline$n$ & 62 & 41 & 15 & 19 & 50 & 46 & \\
\hline Male/female $(n)$ & $42 / 20$ & $28 / 13$ & $9 / 6$ & $13 / 6$ & $38 / 12$ & $34 / 12$ & 0.8 \\
\hline Age (years) & $56 \pm 1.5$ & $48.3 \pm 1.8^{* *}$ & $63.2 \pm 3.1^{*}$ & $56.4 \pm 3.0$ & $58.1 \pm 1.6$ & $58.3 \pm 1.4$ & $<0.0001$ \\
\hline BMI $\left(\mathrm{kg} / \mathrm{m}^{2}\right)$ & $25.7 \pm 0.4$ & $36.7 \pm 0.7 * * *$ & $26.2 \pm 0.8$ & $35.7 \pm 0.9 * * *$ & $26.6 \pm 0.3^{*}$ & $35.5 \pm 0.7 * * *$ & $<0.0001$ \\
\hline $\begin{array}{l}\text { Fasting glucose } \\
(\mathrm{mmol} / \mathrm{l})\end{array}$ & $5.1 \pm 0.1$ & $5.2 \pm 0.2$ & $5.7 \pm 0.1 * * *$ & $6.1 \pm 0.1 * * *$ & $9.9 \pm 0.6^{* * *}$ & $9.6 \pm 0.9^{* * *}$ & $<0.0001^{\mathrm{a}}$ \\
\hline \multicolumn{8}{|l|}{ Lipids (mmol/l) } \\
\hline LDL & $3.6 \pm 0.1$ & $3.3 \pm 0.2$ & $3.1 \pm 0.3$ & $3.8 \pm 0.2$ & $2.9 \pm 0.1 * * *$ & $3.0 \pm 0.1 * * *$ & $<0.0001$ \\
\hline HDL & $1.7 \pm 0.1$ & $1.3 \pm 0.1 * * *$ & $1.5 \pm 0.1$ & $1.2 \pm 0.1 * * *$ & $1.3 \pm 0.1 * * *$ & $1.3 \pm 0.1 * * *$ & $<0.0001$ \\
\hline Triacylglycerol & $1.2 \pm 0.1$ & $1.6 \pm 0.1 * * *$ & $1.3 \pm 0.2$ & $2.0 \pm 0.3 * * *$ & $2.9 \pm 0.9 * * *$ & $2.0 \pm 0.3 * *$ & $0.003^{\mathrm{a}}$ \\
\hline $\begin{array}{l}\text { C-reactive protein } \\
(\mathrm{mg} / \mathrm{l})\end{array}$ & $2.0 \pm 0.2$ & $6.3 \pm 1.1 * * *$ & $6.0 \pm 2.5$ & $4.6 \pm 0.8^{* *}$ & $3.3 \pm 0.4^{* *}$ & $5.3 \pm 0.7 * * *$ & $<0.0001^{\mathrm{a}}$ \\
\hline $\begin{array}{r}\text { Cardiovascular } \\
\text { disease, } n(\%)\end{array}$ & $3(4.8 \%)$ & $7(17.1 \%)$ & $4(26.7 \%)$ & $1(2.3 \%)$ & $10(20 \%)^{*}$ & $10(22 \%)^{* *}$ & 0.06 \\
\hline $\begin{array}{l}\text { Antidepressive } \\
\text { medication, } n(\%)\end{array}$ & $4(6.5 \%)$ & $1(2.5 \%)$ & 0 & $3(15.8 \%)$ & $2(4 \%)$ & $3(6.5 \%)$ & 0.3 \\
\hline Current smoker, $n(\%)$ & $17(27.4 \%)$ & $10(24.4 \%)$ & $2(13.3 \%)$ & $4(21.1 \%)$ & $11(22 \%)$ & $9(19.6 \%)$ & 0.9 \\
\hline
\end{tabular}

Obese BMI $>30$, non-obese BMI $\leq 30 \mathrm{~kg} / \mathrm{m}^{2}$.

Data are means \pm SEM.

${ }^{a}$ Data not normally distributed, statistics performed on log transformed data.

Model for ANOVA: variable $=$ glycaemia/obesity group.

${ }^{*} p=<0.05 ;{ }^{*} p=<0.01 ;{ }^{* *} p=<0.001$ in unpaired $t$ test comparing each of the groups with NGT and no obesity.

zygosity (in all cases $p>0.3$ ). The reduced model included diabetes, obesity status, cholesterol, CRP and the interaction between CRP and diabetes ( $R^{2}$ for the reduced model: $21 \%)$. In the fully adjusted and the reduced model,

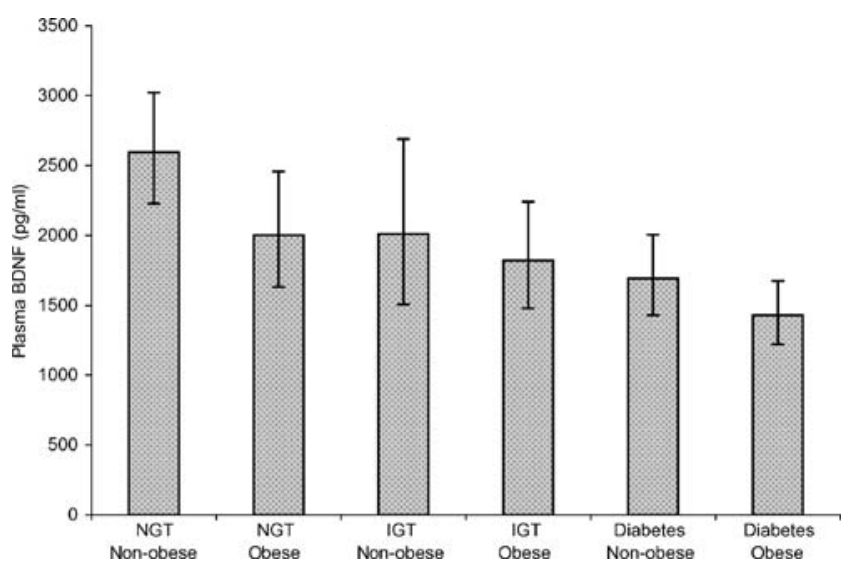

Fig. 1 Plasma concentrations of brain-derived neurotrophic factor $(B D N F)$ are shown for the following six groups: NGT Nonobese $(n=62), N G T$ obese $(n=41), I G T$ non-obese $(n=15), I G T$ obese $(n=19)$, patients with type 2 diabetes, non-obese $(n=50)$ and patients with type 2 diabetes, obese $(n=46)$. Data are expressed as geometric means; error bars represent $95 \%$ CIs. Multiple linear regression (model: $\log [\mathrm{BDNF}]=$ glycaemia group + obesity + glycaemia group $\times$ obesity) showed no interaction between glycaemia group and obesity. A significant effect of glycaemia group $(p<0.0001)$ and of obesity $(p=0.02)$ on plasma BDNF concentrations was observed cholesterols showed a positive association with plasma BDNF ( $p<0.02$ in both models). We also demonstrated a trend towards an interaction between CRP and diabetes $(p<0.1$ in both models) and therefore analysed correlational relationships between BDNF and CRP separately for participants with NGT and for participants with type 2 diabetes. No association between BDNF and CRP was found in participants with NGT, whereas a positive association between BDNF and CRP was seen in the diabetic group in a model also including obesity, CVD, hypertension, cholesterol, and intake of antidepressive medication, statins and fibrates along with age, sex and smoking status $(p=0.002)$.

Plasma BDNF is inversely associated with plasma glucose

Plasma BDNF was inversely associated with HOMA2-IR, which is a measure of insulin resistance, also when adjusted for age, sex and smoking status $(p=0.03)$. A univariate model showed that this association was due to an inverse correlation between plasma BDNF and fasting plasma glucose $(p<0.0001$, Pearson correlation coefficient $=-0.3)$. A multivariate analysis (linear regression model) confirmed the inverse association between plasma BDNF and fasting plasma glucose levels $(p<0.0001)$, whereas insulin had no effect $(p=0.4)$. The association between obesity and BDNF was maintained in this model $(p<0.01)$. 
The 196 BDNF polymorphism is not associated with diabetes

The genotype distributions of BDNF G196A followed Hardy-Weinberg equilibrium. Genotype frequencies did not differ between glycaemia groups. Genotype frequencies in the cohort were: NGT: $G G=69 \%, G A=27 \%$, $\mathrm{AA}=4 \%$; IGT: $50,41,9 \%$; type 2 diabetes: $63,32,5 \%$; overall frequencies: $\mathrm{GG}=64 \%, \mathrm{GA}=31 \%, \mathrm{AA}=5 \%$. Similar results were found when the cohort was further subdivided into glycaemia/obesity subgroups (NGT/nonobese, NGT/obese; IGT/non-obese, IGT/obese; type 2 diabetes/non-obese, type 2 diabetes/obese) (data not shown).

\section{Plasma BDNF output from the human brain}

To determine whether the human brain released BDNF to the circulation, the exchange of BDNF across the brain was studied in seven healthy male volunteers. Since there was no significant change in cerebral perfusion over time and between trials (data not shown), the cerebral output was defined as the jugular-to-arterial concentration difference of BDNF. In resting conditions we found a significant output of BDNF across the brain (Fig. 2a,b), in that the mean AUC for the jugular-toarterial concentration difference in BDNF was different from $0(p=0.02)$.

BDNF output from the brain is abrogated by hyperglycaemia

The finding in the cohort study that plasma BDNF was inversely correlated with plasma glucose raised the possibility that high plasma glucose levels would negatively influence BDNF output. To test the possibility of a direct effect, the above-mentioned seven volunteers were exposed to a hyperglycaemic clamp. When blood glucose levels were maintained at $15 \mathrm{mmol} / \mathrm{l}$ for $3 \mathrm{~h}$, this inhibited BDNF output from the brain (effect of time: $p=0.009$ ) (Fig. 2a).

BDNF output from the brain is not regulated by hyperinsulinaemia

During $3 \mathrm{~h}$ of hyperglycaemia, a gradual increase in plasma insulin levels was observed. To determine if a high plasma insulin level had a direct effect on BDNF output, a euglycaemic-hyperinsulinaemic clamp was performed. At euglycaemic-hyperinsulinaemic clamp conditions, the cerebral output of BDNF was not inhibited (effect of time: $p=$ 0.5 ) (Fig. 2b). Glucose and insulin levels during the two clamps are presented in Table 2.
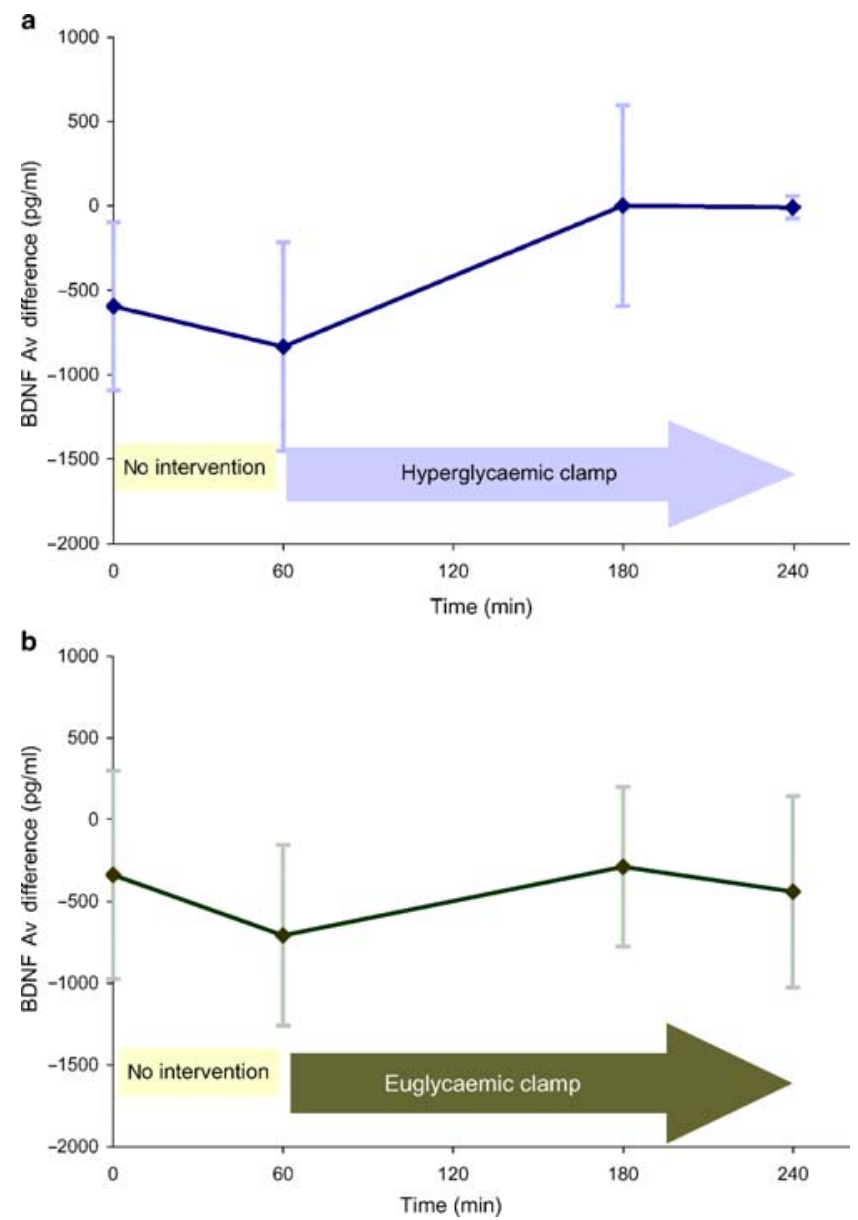

Fig. 2 Differences in plasma BDNF across the brain, measured as jugular-arterial $(A v)$ concentration differences, are shown before and during hyperglycaemic (a) and euglycaemic (b) clamp conditions in seven healthy male subjects. Data are expressed as means; error bars represent 95\% CIs. ANOVA (model: Av difference in BDNF = time; subject was included as a random effect) was used. a Maintaining blood glucose levels at $15 \mathrm{mmol} / \mathrm{l}$ for $3 \mathrm{~h}$ inhibited BDNF output from the brain (effect of time: $p=0.009$ ). At euglycaemic-hyperinsulinaemic clamp conditions (b) the cerebral output of BDNF was not inhibited (effect of time: $p=0.5$ )

\section{Discussion}

In the present study, we demonstrated that there is a cerebral output of BDNF, and that this is inhibited during hyperglycaemic clamp conditions in humans. This may explain the concomitant finding of low circulating levels of BDNF in individuals with type 2 diabetes, and the association between low plasma BDNF and the severity of insulin resistance (HOMA2-IR). We also found a highly significant inverse association between BDNF and plasma glucose, but no association between BDNF and plasma insulin.

In line with an earlier report [23], obesity was associated with lower systemic levels of BDNF in our population. Furthermore, we found no interaction between the effects of type 2 diabetes and obesity on plasma levels of BDNF. The 
Table 2 Plasma glucose and insulin levels during the two experimental days in seven healthy male subjects (Study 2)

\begin{tabular}{|c|c|c|c|c|c|c|c|c|}
\hline \multirow[b]{2}{*}{ Time from clamp initiation $(\mathrm{h})$} & \multicolumn{4}{|c|}{ Hyperglycaemic clamp } & \multicolumn{4}{|c|}{ Euglycaemic clamp } \\
\hline & -1 & 0 & 2 & 3 & -1 & 0 & 2 & 3 \\
\hline Plasma glucose $(\mathrm{mmol} / \mathrm{l})^{\mathrm{a}}$ & - & $4.9 \pm 0.1$ & $14.7 \pm 0.2 *$ & $14.7 \pm 0.3 *$ & - & $5.0 \pm 0.1$ & $5.1 \pm 0.1$ & $4.9 \pm 0.11$ \\
\hline Plasma insulin $(\mathrm{pmol} / \mathrm{l})^{\mathrm{a}}$ & $8 \pm 7$ & $9 \pm 5$ & $517 \pm 81^{*}$ & $688 \pm 100^{*}$ & $10 \pm 8$ & $15 \pm 8$ & $911 \pm 42 *$ & $906 \pm 46^{*}$ \\
\hline
\end{tabular}

Values are means \pm SEM.

${ }^{a}$ Data not normally distributed, statistics performed on log transformed data.

${ }^{*} p=<0.05$ relative to baseline (paired $t$ test).

associations between BDNF on the one hand and obesity and insulin resistance on the other could not be ascribed to antidepressive medication or CVD. Thus our results are in accordance with reports from animal models and with the hypothesis that BDNF plays a role in insulin resistance and in energy balance [7-10].

On the basis of our findings, we suggest that circulating levels of BDNF are regulated in response to plasma levels of glucose. Although the sources of circulating BDNF are not yet positively identified, the brain is a major site of BDNF production, and BDNF crosses the blood-brain barrier in animals [24]. Measurement of cerebral output of BDNF defined as the jugular-to-arterial concentration difference demonstrated output of BDNF from the human brain at basal states. Moreover, in accordance with the cohort data, where high blood glucose was associated with low plasma BDNF, the cerebral output of BDNF was inhibited when blood glucose levels were elevated during clamp conditions. In contrast, the cerebral output of BDNF did not change during hyperinsulinaemic-euglycaemic clamp conditions, indicating that high levels of glucose, but not of insulin, have a negative influence on the output of BDNF from the brain.

The $B D N F$ G196A SNP has been studied for its possible association with Alzheimer's disease and depression, but reports are contradictory [25-29]. In the present study we found no association between this SNP and diabetes or obesity.

Inflammation has been suggested as a key factor in insulin resistance [30]. Systemic low-grade inflammation is defined as two to fourfold increases in circulating levels of inflammatory markers such as CRP [31], and many reports confirm an association between low-grade systemic inflammation on the one hand, and the metabolic syndrome, type 2 diabetes and atherosclerosis on the other [32-37]. Accordingly, it is possible that the association between low levels of BDNF and insulin resistance reflect underlying inflammatory processes. In addition, it has previously been described that patients with acute coronary syndromes have reduced levels of BDNF in plasma [38], which could further point to an association among inflammation, CVD and BDNF. In the present study, high CRP levels were found in participants with type 2 diabetes compared with those without type 2 diabetes, as reported elsewhere [39]. However, no association was found between BDNF and CRP in participants without type 2 diabetes, whereas a positive, rather than inverse, correlation was seen between BDNF and CRP in participants with type 2 diabetes. This indicates that low levels of BDNF are an independent risk factor and not a marker of inflammation. In accordance with this, plasma BDNF levels were not affected when endotoxin was administered to healthy humans (data not shown), whereas CRP increased more than tenfold [40]. In addition, in the present study we found no association between BDNF and CVD or hypertension, although this finding might reflect sample size problems.

Other possible limitations of the study should be considered. The experimental part of this study was performed on healthy male persons and extrapolation to individuals with type 2 diabetes is not necessarily correct. Also, when working with jugular-to-arterial differences, it is not possible to discern whether our findings reflect production by the brain or by the cerebrovascular endothelium. Finally, we cannot exclude the possibility that the entire endothelium, and not only the cerebrovascular endothelium, releases BDNF.

In conclusion, the present study indicates that BDNF plays a role in glucose metabolism and also suggests that neurotrophins such as BDNF may play pathogenetic roles not only in dementia and major depression, but also in type 2 diabetes, potentially explaining the clustering of these diagnoses. The negative correlation between high plasma glucose and the severity of insulin resistance on the one hand and circulating BDNF levels on the other underlines the clinical importance of our study. We also conclude that BDNF is released from the human brain, and that the cerebral output of BDNF is negatively regulated by high plasma glucose levels.

Acknowledgements R. Rousing, H. Villumsen, and K. M. Kristensen are acknowledged for their excellent technical assistance. The Centre of Inflammation and Metabolism is supported by a grant from the Danish National Research Foundation (no. 02-512-55). The study was further supported by the Danish Medical Research Council (52-00-0581), the Novo Nordisk Foundation, the Lundbeck Foundation, the Danish Medical Research Council (no. 22-01-009), the Commission of the European Communities (Contract No. LSHM-CT-2004-005272 EXGENESIS), The Copenhagen Hospital Corporation and The University of Copenhagen. 
Duality of interest The authors declare that they have no duality of interest.

\section{References}

1. Mattson MP, Maudsley S, Martin B (2004) BDNF and 5-HT: a dynamic duo in age-related neuronal plasticity and neurodegenerative disorders. Trends Neurosci 27:589-594

2. Tyler WJ, Alonso M, Bramham CR, Pozzo-Miller LD (2002) From acquisition to consolidation: on the role of brain-derived neurotrophic factor signaling in hippocampal-dependent learning. Learn Mem 9:224-237

3. Tsai SJ (2003) Brain-derived neurotrophic factor: a bridge between major depression and Alzheimer's disease? Med Hypotheses 61:110-113

4. Connor B, Young D, Yan Q, Faull RL, Synek B, Dragunow M (1997) Brain-derived neurotrophic factor is reduced in Alzheimer's disease. Brain Res Mol Brain Res 49:71-81

5. Laske C, Stransky E, Leyhe T et al (2006) Stage-dependent BDNF serum concentrations in Alzheimer's disease. J Neural Transm 113:1217-1224

6. Karege F, Perret G, Bondolfi G, Schwald M, Bertschy G, Aubry JM (2002) Decreased serum brain-derived neurotrophic factor levels in major depressed patients. Psychiatry Res 109:143-148

7. Ono M, Ichihara J, Nonomura T et al (1997) Brain-derived neurotrophic factor reduces blood glucose level in obese diabetic mice but not in normal mice. Biochem Biophys Res Commun 238:633-637

8. Tonra JR, Ono M, Liu X et al (1999) Brain-derived neurotrophic factor improves blood glucose control and alleviates fasting hyperglycemia in C57BLKS-Lepr(db)/lepr(db) mice. Diabetes 48:588-594

9. Nakagawa T, Tsuchida A, Itakura Y et al (2000) Brain-derived neurotrophic factor regulates glucose metabolism by modulating energy balance in diabetic mice. Diabetes 49:436-444

10. Kernie SG, Liebl DJ, Parada LF (2000) BDNF regulates eating behavior and locomotor activity in mice. EMBO J 19:1290-1300

11. Cotman CW (2005) The role of neurotrophins in brain aging: a perspective in honor of Regino Perez-Polo. Neurochem Res 30:877-881

12. Strachan MW, Deary IJ, Ewing FM, Frier BM (1997) Is type II diabetes associated with an increased risk of cognitive dysfunction? A critical review of published studies. Diabetes Care $20: 438-445$

13. Awad N, Gagnon M, Messier C (2004) The relationship between impaired glucose tolerance, type 2 diabetes, and cognitive function. J Clin Exp Neuropsychol 26:1044-1080

14. Allen KV, Frier BM, Strachan MW (2004) The relationship between type 2 diabetes and cognitive dysfunction: longitudinal studies and their methodological limitations. Eur J Pharmacol 19 (490):169-175

15. Biessels GJ, Staekenborg S, Brunner E, Brayne C, Scheltens P (2006) Risk of dementia in diabetes mellitus: a systematic review. Lancet Neurol 5:64-74

16. Anderson RJ, Freedland KE, Clouse RE, Lustman PJ (2001) The prevalence of comorbid depression in adults with diabetes: a meta-analysis. Diabetes Care 24:1069-1078

17. Hassing LB, Hofer SM, Nilsson SE et al (2004) Comorbid type 2 diabetes mellitus and hypertension exacerbates cognitive decline: evidence from a longitudinal study. Age Ageing 33:355-361

18. Kanaya AM, Barrett-Connor E, Gildengorin G, Yaffe K (2004) Change in cognitive function by glucose tolerance status in older adults: a 4-year prospective study of the Rancho Bernardo study cohort. Arch Intern Med 164:1327-1333

19. Bruce DG, Davis WA, Starkstein SE, Davis TM (2005) A prospective study of depression and mortality in patients with type 2 diabetes: The Fremantle Diabetes Study. Diabetologia 48:2532-2539
20. Egan MF, Kojima M, Callicott JH et al (2003) The BDNF val66met polymorphism affects activity-dependent secretion of BDNF and human memory and hippocampal function. Cell 112:257-269

21. Matthews DR, Hosker JP, Rudenski AS, Naylor BA, Treacher DF, Turner RC (1985) Homeostasis model assessment: insulin resistance and beta-cell function from fasting plasma glucose and insulin concentrations in man. Diabetologia 28:412-419

22. Wallace TM, Levy JC, Matthews DR (2004) Use and abuse of HOMA modeling. Diabetes Care 27:1487-1495

23. Lommatzsch M, Zingler D, Schuhbaeck K et al (2005) The impact of age, weight and gender on BDNF levels in human platelets and plasma. Neurobiol Aging 26:115-123

24. Pan W, Banks WA, Fasold MB, Bluth J, Kastin AJ (1998) Transport of brain-derived neurotrophic factor across the bloodbrain barrier. Neuropharmacology 37:1553-1561

25. Li Y, Rowland C, Tacey K et al (2005) The BDNF Val66Met polymorphism is not associated with late onset Alzheimer's disease in three case-control samples. Mol Psychiatry 10:809-810

26. Surtees PG, Wainwright NW, Willis-Owen SA et al (2006) No association between the BDNF Val66Met polymorphism and mood status in a non-clinical community sample of 7389 older adults. J Psychiatr Res (in press). DOI 10.1016/j.jpsychires.2006.05.015

27. Hwang JP, Tsai SJ, Hong CJ, Yang CH, Lirng JF, Yang YM (2006) The Val66Met polymorphism of the brain-derived neurotrophic-factor gene is associated with geriatric depression. Neurobiol Aging 27:1834-1837

28. Schumacher J, Jamra RA, Becker T et al (2005) Evidence for a relationship between genetic variants at the brain-derived neurotrophic factor (BDNF) locus and major depression. Biol Psychiatry 58:307-314

29. Matsushita S, Arai $H$, Matsui $T$ et al (2005) Brain-derived neurotrophic factor gene polymorphisms and Alzheimer's disease. J Neural Transm 112:703-711

30. Dandona P, Aljada A, Bandyopadhyay A (2004) Inflammation: the link between insulin resistance, obesity and diabetes. Trends Immunol 25:4-7

31. Bruunsgaard H (2005) Physical activity and modulation of systemic low-level inflammation. J Leukoc Biol 78:819-835

32. Barzilay JI, Abraham L, Heckbert SR et al (2001) The relation of markers of inflammation to the development of glucose disorders in the elderly: the Cardiovascular Health Study. Diabetes 50:2384-2389

33. Duncan BB, Schmidt MI, Pankow JS et al (2003) Low-grade systemic inflammation and the development of type 2 diabetes: the atherosclerosis risk in communities study. Diabetes 52:1799-1805

34. Festa A, D'Agostino R Jr, Tracy RP, Haffner SM (2002) Elevated levels of acute-phase proteins and plasminogen activator inhibitor1 predict the development of type 2 diabetes: the insulin resistance atherosclerosis study. Diabetes 51:1131-1137

35. Freeman DJ, Norrie J, Caslake MJ et al (2002) C-reactive protein is an independent predictor of risk for the development of diabetes in the West of Scotland Coronary Prevention Study. Diabetes 51:1596-1600

36. Han TS, Sattar N, Williams K, Gonzalez-Villalpando C, Lean ME, Haffner SM (2002) Prospective study of C-reactive protein in relation to the development of diabetes and metabolic syndrome in the Mexico City Diabetes Study. Diabetes Care 25:2016-2021

37. Pradhan AD, Manson JE, Rifai N, Buring JE, Ridker PM (2001) C-reactive protein, interleukin 6 , and risk of developing type 2 diabetes mellitus. JAMA 286:327-334

38. Manni L, Nikolova V, Vyagova D, Chaldakov GN, Aloe L (2005) Reduced plasma levels of NGF and BDNF in patients with acute coronary syndromes. Int J Cardiol 102:169-171

39. Ford ES (1999) Body mass index, diabetes, and C-reactive protein among US adults. Diabetes Care 22:1971-1977

40. Krabbe KS, Bruunsgaard H, Hansen CM et al (2001) Ageing is associated with a prolonged fever response in human endotoxemia. Clin Diagn Lab Immunol 8:333-338 